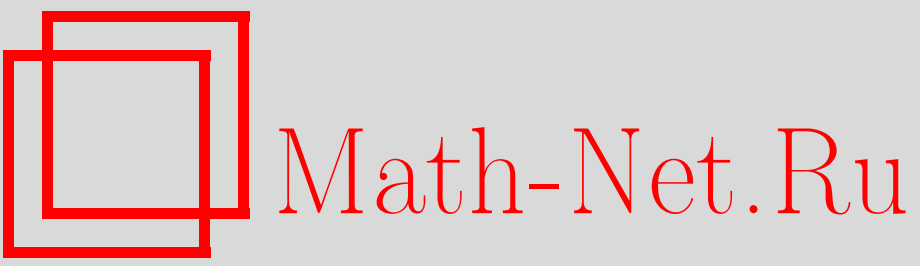

М. А. Королёв, О больших значениях функции $S(t)$ на коротких промежутках, Изв. РАН. Сер. матем., 2005, том 69, выпуск 1, 115-124

DOI: https://doi.org/10.4213/im625

Использование Общероссийского математического портала Math-Net.Ru подразумевает, что вы прочитали и согласны с пользовательским соглашением

http: //www. mathnet.ru/rus/agreement

Параметры загрузки:

IP : 54.196 .121 .252

26 апреля 2023 г., $17: 15: 44$ 
УДК 511

\author{
М. А. Королёв
}

\title{
О больших значениях функции $S(t)$ на коротких промежутках
}

\author{
Изучаются верхняя и нижняя грани аргумента дзета-функции Римана на ко- \\ ротких промежутках критической прямой. \\ Библиография: 15 наименований.
}

\section{§1. Введение}

В настоящей работе продолжены исследования свойств аргумента дзета-функции Римана $\zeta(s)$ на критической прямой $\operatorname{Re} s=1 / 2$, начатые в [1]-[7].

Известно, что функция $S(t)=\pi^{-1} \arg \zeta(1 / 2+i t)$ при $t \rightarrow+\infty$ меняет знак бесконечно много раз (см. [8, с. 222]), но в то же время может принимать сколь угодно большие по абсолютной величине как положительные, так и отрицательные значения.

В 1946 г. А. Сельберг [9] доказал неравенства

$$
\sup _{T \leqslant t \leqslant 2 T}( \pm S(t)) \geqslant A \frac{(\ln T)^{1 / 3}}{(\ln \ln T)^{7 / 3}}
$$

в которых $A$ - положительная абсолютная постоянная. Один из возможных путей уточнения этих оценок состоит в замене правых частей неравенств (1) бо́льшими величинами.

Так, в 1977 г. Х. Монтгомери [10], пользуясь гипотезой Римана, установил сушествование на любом промежутке $\left(T^{1 / 6}, T\right)$ точек $t_{0}$ и $t_{1}$, для которых

$$
(-1)^{j} S\left(t_{j}\right) \geqslant \frac{1}{20} \sqrt{\frac{\ln T}{\ln \ln T}}, \quad j=0,1 .
$$

В 1986 г. К.-М. Тсанг [11], развивая метод работы [9], усилил результаты А. Сельберга и Х. Монтгомери и получил неравенства

$$
\sup _{T \leqslant t \leqslant 2 T}( \pm S(t)) \geqslant A\left(\frac{\ln T}{\ln \ln T}\right)^{a},
$$

в которых $A$ - абсолютная постоянная, $A>0$, а величина $a$ берется равной $1 / 2$, если гипотеза Римана верна, и равной $1 / 3$ в противном случае.

Наилучшая из верхних оценок $|S(t)|$, опирающаяся на гипотезу Римана, принадлежит К. Рамачандре и А. Санкаранарайанану [12]:

$$
|S(t)|<1.2 \frac{\ln t}{\ln \ln t}, \quad t>t_{0}>0 .
$$


Предполагается, однако, что окончательный результат имеет вид

$$
S(t)=O\left((\ln t)^{a}\right)
$$

где $a=1 / 2+o(1)$ при $t \rightarrow+\infty$ (см. [10], [13, с.21]). В пользу этой гипотезы свидетельствует и условная теорема Я. Мозера [14], согласно которой на всяком интервале $(T, 2 T)$ существует не менее $c T \ln T$ промежутков $\left[\gamma, \gamma^{\prime}\right)$ таких, что

$$
|S(t)|<c_{1}(\ln T)^{1 / 2}(\ln \ln T)^{2 / 3}, \quad \gamma \leqslant t \leqslant \gamma^{\prime} .
$$

Здесь $c, c_{1}$ - положительные абсолютные постоянные, а $\gamma, \gamma^{\prime}$ - последовательные ординаты комплексных нулей $\zeta(s)$.

Иной путь уточнения неравенств (1)-(3) состоит в замене промежутка $(T, 2 T)$, на котором изучаются верхняя и нижняя грани $S(t)$, на более короткий промежуток $(T, T+H), 0<H<T$. Доказательству подобного утверждения и посвящена настоящая работа. Основным результатом статьи является

Tеорема 1. Пусть $T>T_{0}>0,(\ln T)(\ln \ln T)^{-3 / 2}<H<T$. Если гипотеза Римана верна, то справедливы неравенства

$$
\sup _{T-H \leqslant t \leqslant T+2 H}( \pm S(t)) \geqslant \frac{1}{90 \pi} \sqrt{\frac{\ln H}{\ln \ln H}} .
$$

Доказательство этой теоремы основано на лемме 1, которая представляет собой частный случай одного утверждения из работы [11]. Согласно этой лемме выполнение для некоторых $T, H, M$ и $k(H, M>0, k \geqslant 1)$ и вешественной функции $W(t)$ неравенств

$$
\int_{T}^{T+H}(W(t))^{2 k} d t \geqslant H M^{2 k}, \quad\left|\int_{T}^{T+H}(W(t))^{2 k+1} d t\right| \leqslant \frac{1}{2} H M^{2 k+1}
$$

влечет существование на промежутке $(T, T+H)$ точек $t_{0}$ и $t_{1}$, в которых $W\left(t_{0}\right) \geqslant$ $0.5 M, W\left(t_{1}\right) \leqslant-0.5 M$.

В рассматриваемой задаче роль $W(t)$ играет тригонометрический полином

$$
-\sum_{p \leqslant e^{\tau}} \frac{\sin (t \ln p)}{\sqrt{p}}\left(1-\frac{\ln p}{\tau}\right), \quad \tau>0
$$

Соотношение, связывающее $W(t)$ с $S(t)$, впервые было установлено А. Сельбергом в [9], а его обобщение было получено К.-М. Тсангом [11]. В настоящей статье доказательству этого соотношения посвяшена лемма 2.

Оригинальной частью работы является лемма 3 , устанавливающая оптимальные значения величин $k$ и $M$, при которых полином $W(t)$ удовлетворяет неравенствам (5) при $H>(\ln T)(\ln \ln T)^{-3 / 2}$. Лемма 3 содержит, в частности, новый способ доказательства первого из соотношений (5).

Следствием основной теоремы является следующее утверждение, доказательство которого также приведено в статье. 
Tеорема 2. Пусть $\varepsilon$ - сколь угодно малое фиксированное число, $0<\varepsilon<1$, $T>T_{0}(\varepsilon)>0$, и пусть $E_{j}, j=0,1,-$ множество значений $t, T \leqslant t \leqslant 2 T$, для которьх

Тогда справедливы неравенства

$$
(-1)^{j} S(t)>\frac{\sqrt{\varepsilon}}{300} \sqrt{\frac{\ln T}{\ln \ln T}} .
$$

$$
\operatorname{mes}\left(E_{j}\right)>\frac{1}{3} T^{1-\varepsilon}(\ln T)^{-1}, \quad j=0,1 .
$$

Отметим, что этот результат не следует из теорем А. Гоша [15] о распределении значений $S(t)$.

\section{§2. Вспомогательные леммы}

В настоящем параграфе приводятся вспомогательные утверждения, необходимые для доказательства теорем 1 и 2 .

Лемма 1. Пусть $H>0, M>0, k$-иелое, $k \geqslant 1$, и пусть вещественная непрерьвная функщия $W(t)$ удовлетворяет условиям

$$
\int_{T}^{T+H}(W(t))^{2 k} d t \geqslant H M^{2 k}, \quad\left|\int_{T}^{T+H}(W(t))^{2 k+1} d t\right| \leqslant \frac{1}{2} H M^{2 k+1} .
$$

Тогда справедливы неравенства

$$
\max _{T \leqslant t \leqslant T+H} W(t) \geqslant \frac{1}{2} M, \quad \min _{T \leqslant t \leqslant T+H} W(t) \leqslant-\frac{1}{2} M .
$$

ДОКАЗАТЕЛЬСТВО. Положим

так что

$$
W_{r}(t)=\frac{1}{2}\left(|W(t)|+(-1)^{r} W(t)\right),
$$

$$
W_{0}(t)=\max (0, W(t)), \quad W_{1}(t)=|\min (0, W(t))|
$$

И

$$
\begin{aligned}
& \int_{T}^{T+H}\left(W_{r}(t)\right)^{2 k+1} d t \\
& \quad=\frac{1}{2}\left(\int_{T}^{T+H}|W(t)|^{2 k+1} d t+(-1)^{r} \int_{T}^{T+H}(W(t))^{2 k+1} d t\right), \quad r=0,1 .
\end{aligned}
$$

Согласно неравенству Гёльдера имеем

откуда

$$
H M^{2 k} \leqslant \int_{T}^{T+H}|W(t)|^{2 k} d t \leqslant H^{\frac{1}{2 k+1}}\left(\int_{T}^{T+H}|W(t)|^{2 k+1} d t\right)^{1-\frac{1}{2 k+1}},
$$

$$
\begin{gathered}
\int_{T}^{T+H}|W(t)|^{2 k+1} d t \geqslant H M^{2 k+1} \\
\int_{T}^{T+H}\left(W_{r}(t)\right)^{2 k+1} d t \geqslant \frac{1}{2}\left(H M^{2 k+1}-\frac{1}{2} H M^{2 k+1}\right) \geqslant H\left(\frac{1}{2} M\right)^{2 k+1}, \quad r=0,1 .
\end{gathered}
$$

Поскольку функции $W_{0}(t)$ и $W_{1}(t)$ неотрицательны, то из последнего неравенства следует, что каждая из них принимает значения, превосходящие $\frac{1}{2} M$ хотя бы в одной точке промежутка $(T, T+H)$. Лемма доказана. 
Лемма 2. Если гипотеза Римана верна, то для любых достаточно больших положительных $H, t u \tau, \tau<\ln t, H<t$, справедлива формула

$$
\int_{-H \tau / 2}^{H \tau / 2}\left(\frac{\sin u}{u}\right)^{2} S\left(t+\frac{2 u}{\tau}\right) d u=W(t)+O\left(\ln \tau+\frac{1}{H \tau} \frac{\ln t}{\ln \ln t}\right)
$$

əде

$$
W(t)=-\sum_{p \leqslant e^{\tau}} \frac{\sin (t \ln p)}{\sqrt{p}}\left(1-\frac{\ln p}{\tau}\right),
$$

а постоянная в знаке $O-$ абсолютная.

ДоКАЗАТЕЛЬСТВо. Воспользуемся следующей формулой, доказанной в [11]:

$$
\begin{aligned}
& \int_{-\infty}^{+\infty} f(u) \ln \zeta\left(\frac{1}{2}+i(t+u)\right) d u=\sum_{n=2}^{+\infty} \frac{\Lambda(n)}{\ln n} \frac{n^{-i t}}{\sqrt{n}} \hat{f}(\ln n) \\
& \quad+2 \pi\left(\sum_{\beta} \int_{0}^{\beta-\frac{1}{2}} f(\gamma-t-i \xi) d \xi-\int_{0}^{\frac{1}{2}} f(-t-i \xi) d \xi\right) .
\end{aligned}
$$

Здесь $f(z)$ - функция, вещественная на вещественной прямой, аналитическая в полосе $|\operatorname{Im} z| \leqslant 1$ и удовлетворяющая в этой полосе неравенству $|f(z)| \ll(|z|+1)^{-a}$, $a>1$,

$$
\hat{f}(x)=\int_{-\infty}^{+\infty} f(u) e^{-i x u} d u
$$

а суммирование ведется по всем нулям $\varrho=\beta+i \gamma$ дзета-функции Римана, лежащим правее критической прямой $(\beta>1 / 2)$. Если гипотеза Римана верна, то эта сумма будет равна нулю.

Пусть

$$
f(z)=\left(\frac{\sin \frac{\tau z}{2}}{\frac{z}{2}}\right)^{2}
$$

Тогда

$$
\begin{aligned}
\hat{f}(x) & =\int_{-\infty}^{+\infty} e^{-i x u}\left(\frac{\sin \frac{\tau u}{2}}{\frac{u}{2}}\right)^{2} d u=2 \tau \int_{-\infty}^{+\infty} e^{-i \xi \frac{2 x}{\tau}}\left(\frac{\sin \xi}{\xi}\right)^{2} d \xi \\
& =2 \tau \frac{\pi}{2} \max \left(0,2-2\left|\frac{x}{\tau}\right|\right)=2 \pi \tau \max \left(0,1-\left|\frac{x}{\tau}\right|\right), \\
\hat{f}(\ln n) & = \begin{cases}2 \pi \tau\left(1-\frac{\ln n}{\tau}\right), & \text { если } 1 \leqslant n \leqslant e^{\tau}, \\
0, & \text { если } n>e^{\tau} .\end{cases}
\end{aligned}
$$

Преобразуя в исходном равенстве мнимую часть, будем иметь

$$
\begin{gathered}
\pi \int_{-\infty}^{+\infty} f(u) S(t+u) d u=-2 \pi \tau \sum_{n \leqslant e^{\tau}} \frac{\Lambda(n)}{\ln n} \frac{\sin (t \ln n)}{\sqrt{n}}\left(1-\frac{\ln n}{\tau}\right) \\
-2 \pi \operatorname{Im} \int_{0}^{\frac{1}{2}}\left(\frac{\sin \frac{\tau}{2}(t+i \xi)}{\frac{1}{2}(t+i \xi)}\right)^{2} d \xi
\end{gathered}
$$


Поскольку при $0 \leqslant \xi \leqslant 1 / 2$

$$
\left|\frac{\sin \frac{\tau}{2}(t+i \xi)}{\frac{1}{2}(t+i \xi)}\right|^{2} \leqslant\left(\frac{2 e^{\tau \xi / 2}}{t}\right)^{2} \leqslant\left(\frac{2 e^{\tau / 4}}{t}\right)^{2}<\frac{4 e^{\frac{1}{2} \ln t}}{t^{2}}<0.1
$$

то последний интеграл по абсолютной величине не превосходит $0.1 \cdot 2 \pi$.

В сумме по $n$ выделим слагаемые, отвечающие простым $n=p$, и оценим оставшиеся по модулю:

$$
\begin{aligned}
& \left|\sum_{k \geqslant 2} \sum_{n=p^{k} \leqslant e^{\tau}} \frac{\Lambda(n)}{\ln n} \frac{\sin (t \ln n)}{\sqrt{n}}\left(1-\frac{\ln n}{\tau}\right)\right| \\
& \quad \leqslant \frac{1}{2} \sum_{p \leqslant e^{\tau / 2}} \sum_{k \geqslant 2} p^{-k / 2}=\frac{1}{2} \sum_{p \leqslant e^{\tau / 2}} \frac{1}{p} \frac{1}{1-p^{-1 / 2}} \\
& \quad<2 \sum_{p \leqslant e^{\tau / 2}} \frac{1}{p}=2\left(\ln \frac{\tau}{2}+0.26 \ldots+O\left(\tau^{-1}\right)\right)<2 \ln \tau-0.1 .
\end{aligned}
$$

Таким образом, исходная формула принимает вид

$$
\begin{aligned}
& \pi \int_{-\infty}^{+\infty}\left(\frac{\sin \frac{\tau u}{2}}{\frac{u}{2}}\right)^{2} S(t+u) d u=-2 \pi \tau \sum_{p \leqslant e^{\tau}} \frac{\sin (t \ln p)}{\sqrt{p}}\left(1-\frac{\ln p}{\tau}\right) \\
& \quad+2 \theta \pi \tau(2 \ln \tau-0.1+0.1), \quad|\theta| \leqslant 1 .
\end{aligned}
$$

Делая в интеграле замену $v=\frac{\tau u}{2}$ и сокращая обе части последнего равенства на $2 \pi \tau$, находим

$$
\int_{-\infty}^{+\infty}\left(\frac{\sin v}{v}\right)^{2} S\left(t+\frac{2 v}{\tau}\right) d v=-\sum_{p \leqslant e^{\tau}} \frac{\sin (t \ln p)}{\sqrt{p}}\left(1-\frac{\ln p}{\tau}\right)+2 \theta \ln \tau .
$$

Для завершения доказательства представим левую часть в виде суммы:

$$
\left(\int_{-H \tau / 2}^{H \tau / 2}+\int_{-\infty}^{-H \tau / 2}+\int_{H \tau / 2}^{+\infty}\right)\left(\frac{\sin v}{v}\right)^{2} S\left(t+\frac{2 v}{\tau}\right) d v
$$

и оценим последние два интеграла, пользуясь неравенством (4):

$$
\begin{aligned}
& \left|\left(\int_{-\infty}^{-H \tau / 2}+\int_{H \tau / 2}^{+\infty}\right)\left(\frac{\sin v}{v}\right)^{2} S\left(t+\frac{2 v}{\tau}\right) d v\right| \ll \tau^{-1} \int_{H}^{+\infty} \frac{1}{v^{2}} \frac{\ln (t+v)}{\ln \ln (t+v)} d v \\
& \quad \ll \tau^{-1} \int_{H}^{t} \frac{1}{v^{2}} \frac{\ln t}{\ln \ln t} d v+\tau^{-1} \int_{t}^{+\infty} \frac{1}{v^{3 / 2}} \frac{\ln v}{\sqrt{v} \ln \ln v} d v \\
& \quad \ll \frac{1}{H \tau} \frac{\ln t}{\ln \ln t}+\frac{1}{t \tau} \frac{\ln t}{\ln \ln t} \ll \frac{1}{H \tau} \frac{\ln t}{\ln \ln t} .
\end{aligned}
$$

Лемма доказана. 
ЛЕмма 3. Пусть $T>T_{0}>0,(\ln T)(\ln \ln T)^{-3 / 2}<H<T$. Тогда

$$
\max _{T \leqslant t \leqslant T+H} W(t) \geqslant \frac{1}{170} \sqrt{\frac{\ln H}{\ln \ln H}}, \quad \min _{T \leqslant t \leqslant T+H} W(t) \leqslant-\frac{1}{170} \sqrt{\frac{\ln H}{\ln \ln H}} .
$$

ДокАЗАТЕЛЬСТво. Пусть $k$ - достаточно большое натуральное число, точное значение которого будет выбрано позже, и

$$
I_{k}=\int_{T}^{T+H}(W(t))^{2 k} d t, \quad J_{k}=\int_{T}^{T+H}(W(t))^{2 k+1} d t
$$

Представляя $W(t)$ в виде разности:

$$
W(t)=\frac{V(t)-\bar{V}(t)}{2 i}
$$

где

$$
V(t)=\sum_{p \leqslant e^{\tau}} \frac{a(p)}{\sqrt{p}} p^{-i t}, \quad a(p)=1-\frac{\ln p}{\tau}
$$

находим

$$
\begin{aligned}
& I_{k}=(2 i)^{-2 k} \sum_{\nu=0}^{2 k}(-1)^{\nu}\left(\begin{array}{c}
2 k \\
\nu
\end{array}\right) j_{2 k}(\nu) \\
& J_{k}=(2 i)^{-2 k-1} \sum_{\nu=0}^{2 k+1}(-1)^{\nu}\left(\begin{array}{c}
2 k+1 \\
\nu
\end{array}\right) j_{2 k+1}(\nu)
\end{aligned}
$$

здесь

$$
\begin{aligned}
j_{r}(\nu) & =\int_{T}^{T+H}(V(t))^{\nu}(\bar{V}(t))^{r-\nu} d t \\
& =\sum_{p_{1}, \ldots, p_{\nu} \leqslant e^{\tau}} \sum_{q_{1}, \ldots, q_{r-\nu} \leqslant e^{\tau}} \frac{a\left(p_{1}\right) \ldots a\left(q_{r-\nu}\right)}{\sqrt{p_{1} \ldots q_{r-\nu}}} \int_{T}^{T+H}\left(\frac{q_{1} \ldots q_{r-\nu}}{p_{1} \ldots p_{\nu}}\right)^{i t} d t .
\end{aligned}
$$

Из основной теоремы арифметики следует, что равенство $q_{1} \ldots q_{r-\nu}=p_{1} \ldots p_{\nu}$ возможно лишь в случае, когда $r=2 k, \nu=k$. В остальных случаях, полагая для краткости $Q=q_{1} \ldots q_{r-\nu}, P=p_{1} \ldots p_{\nu}$ и замечая, что $P, Q \leqslant e^{r \tau}$, находим

$$
\begin{aligned}
\left|j_{r}(\nu)\right| & \leqslant \sum_{P \neq Q} \frac{1}{\sqrt{P Q}} 2\left|\ln \frac{Q}{P}\right|^{-1} \leqslant 2 \sum_{P, Q} \frac{1}{\sqrt{P Q}} \max (P, Q) \\
& \leqslant 2 e^{r \tau} \sum_{P, Q} \frac{1}{\sqrt{P Q}} \leqslant 2 e^{r \tau}\left(\sum_{p \leqslant e^{\tau}} \frac{1}{\sqrt{p}}\right)^{r} \leqslant 2 e^{r \tau}\left(\frac{3 e^{\tau / 2}}{\tau}\right)^{r}<e^{3 r \tau / 2} .
\end{aligned}
$$


В случае $r=2 k, \nu=k$ в выражении для $j_{r}(\nu)$ присутствуют "диагональные" члены:

$$
\begin{aligned}
j_{2 k}(k) & =H \Sigma+\theta \sum_{P \neq Q} \frac{1}{\sqrt{P Q}} 2\left|\ln \frac{Q}{P}\right|^{-1}=H \Sigma+\theta e^{\frac{3}{2} 2 k \tau}, \quad|\theta| \leqslant 1, \\
\Sigma & =\sum_{p_{1} \ldots p_{k}=q_{1} \ldots q_{k}} \frac{a\left(p_{1}\right) \ldots a\left(q_{k}\right)}{\sqrt{p_{1} \ldots q_{k}}}=\sum_{p_{1} \ldots p_{k}=q_{1} \ldots q_{k}} \frac{a^{2}\left(p_{1}\right) \ldots a^{2}\left(p_{k}\right)}{p_{1} \ldots p_{k}} .
\end{aligned}
$$

Оставляя в последней сумме слагаемые, отвечающие наборам $\left(p_{1}, \ldots, p_{k}\right)$, не содержашим повторений, придем к нижней оценке величины $\Sigma$. Зафиксируем набор $\left(p_{1}, \ldots, p_{k}\right)$, в котором все числа $p_{1}, \ldots, p_{k}$ различны. Тогда число решений уравнения $p_{1} \ldots p_{k}=q_{1} \ldots q_{k}$ в простых числах $q_{1}, \ldots, q_{k}$ равно $k !$, и поэтому

$$
\Sigma \geqslant k ! \sum_{p_{1} \leqslant e^{\tau}} \frac{a^{2}\left(p_{1}\right)}{p_{1}} \sum_{\substack{p_{2} \leqslant e^{\tau} \\ p_{2} \neq p_{1}}} \frac{a^{2}\left(p_{2}\right)}{p_{2}} \sum_{\substack{p_{3} \leqslant e^{\tau} \\ p_{3} \neq p_{1}, p_{2}}} \frac{a^{2}\left(p_{3}\right)}{p_{3}} \ldots \sum_{\substack{p_{k} \leqslant e^{\tau} \\ p_{k} \neq p_{1}, p_{2}, \ldots, p_{k-1}}} \frac{a^{2}\left(p_{k}\right)}{p_{k}} .
$$

Величины $a^{2}(p) / p$ с ростом $p, p<e^{\tau}$, монотонно убывают, так как

$$
\frac{d}{d p}\left(\frac{a^{2}(p)}{p}\right)=\frac{d}{d p}\left(\frac{1}{p}\left(1-\frac{\ln p}{\tau}\right)^{2}\right)=-\frac{1}{p^{2}}\left(1-\frac{\ln p}{\tau}\right)\left(1-\frac{\ln p}{\tau}+\frac{2}{\tau}\right)<0 .
$$

Следовательно, при любых значениях $p_{1}, \ldots, p_{k-1}$ сумма по $p_{k}$ не меньше суммы величин $a^{2}(p) / p$ по простым $p \leqslant e^{\tau}$, из которой исключены слагаемые, отвечающие первым $k-1$ простым числам. Считая $k$ и $\tau$ такими, что $(k-1)$-е простое число не превосходит $2 k \ln k$ и $2 k \ln k<e^{2 \tau / 3}$, убеждаемся, что сумма по $p_{k}$ оценивается снизу величиной

$$
\sum_{e^{2 \tau / 3}<p \leqslant e^{\tau}} \frac{a^{2}(p)}{p}
$$

которая не зависит от $p_{1}, \ldots, p_{k-1}$. Применим те же рассуждения к сумме по $p_{k-1}$, затем к суммам по $p_{k-2}, \ldots, p_{1}$. Таким образом, получим

$$
\begin{aligned}
\Sigma & \geqslant k !\left(\sum_{e^{2 \tau / 3}<p \leqslant e^{\tau}} \frac{a^{2}(p)}{p}\right)^{k}>k !\left(\sum_{e^{2 \tau / 3}<p \leqslant e^{4 \tau / 5}} \frac{1}{p}\left(1-\frac{\ln p}{\tau}\right)^{2}\right)^{k} \\
& >k !\left(\sum_{e^{2 \tau / 3}<p \leqslant e^{4 \tau / 5}} \frac{1}{5^{2} p}\right)^{k}=5^{-2 k} k !\left(\ln \frac{4}{5} \tau-\ln \frac{2}{3} \tau+o(1)\right)^{k} \\
& >(0.1)^{k} 5^{-2 k} k !=(0.004)^{k} k ! .
\end{aligned}
$$

Итак,

$$
\begin{gathered}
j_{2 k}(k)>(0.004)^{k} k ! H-e^{3 k \tau}, \\
I_{k}>2^{-2 k}\left(\begin{array}{c}
2 k \\
k
\end{array}\right)(0.004)^{k} k ! H-e^{3 k \tau} 2^{-2 k} \sum_{\nu=0}^{2 k}\left(\begin{array}{c}
2 k \\
\nu
\end{array}\right)>\left(0.004 e^{-1} k\right)^{k} H-e^{3 k \tau}, \\
\left|J_{k}\right|<2^{-2 k-1} e^{\frac{3}{2}(2 k+1) \tau} \sum_{\nu=0}^{2 k+1}\left(\begin{array}{c}
2 k+1 \\
\nu
\end{array}\right)=e^{3 k \tau} e^{\frac{3 \tau}{2}}
\end{gathered}
$$


Положим

$$
\tau=2 \ln \ln H, \quad k=\left[\frac{\ln H}{7 \ln \ln H}\right] .
$$

Тогда

$$
\begin{gathered}
2 k \ln k<2\left(\frac{\ln H}{7 \ln \ln H}+1\right) \ln \ln H<(\ln H)^{4 / 3}=e^{2 \tau / 3}, \\
e^{3 k \tau}<\exp \left(6 \ln \ln H\left(\frac{\ln H}{7 \ln \ln H}+1\right)\right)=H^{6 / 7}(\ln H)^{6}, \\
I_{k}>\left(0.004 e^{-1} k\right)^{k} H-H^{6 / 7}(\ln H)^{6}>(0.001 k)^{k} H \\
\left|J_{k}\right|<H^{6 / 7}(\ln H)^{9}<\frac{1}{2}(0.001 k)^{k} H .
\end{gathered}
$$

Таким образом, для функции $W(t)$ выполнены условия леммы 1 при $M=\sqrt{0.001 k}$, $k=\left[\frac{\ln H}{7 \ln \ln H}\right]$. Следовательно, на отрезке $T \leqslant t \leqslant T+H$ найдутся такие точки $t_{0}$ и $t_{1}$, что $W\left(t_{0}\right) \geqslant \frac{1}{2} M, W\left(t_{1}\right) \leqslant-\frac{1}{2} M$.

Для завершения доказательства осталось заметить, что

$$
\frac{1}{2} M=\frac{1}{2} \sqrt{0.001 k}>\frac{1}{170} \sqrt{\frac{\ln H}{\ln \ln H}} .
$$

Лемма доказана.

\section{§3. Доказательства теорем 1 и 2}

ДОКАЗАТЕЛЬСТВО ТЕОРЕмЫ 1 . Пусть $\tau=2 \ln \ln H$. Согласно леммам 2 и 3 на отрезке $T \leqslant t \leqslant T+H$ найдутся точки $t_{0}$ и $t_{1}$, для которых

$$
\begin{aligned}
& j_{0}=\int_{-H \tau / 2}^{H \tau / 2}\left(\frac{\sin u}{u}\right)^{2} S\left(t_{0}+\frac{2 u}{\tau}\right) d u \geqslant \frac{1}{170} \sqrt{\frac{\ln H}{\ln \ln H}}+O\left(\ln \tau+\frac{1}{H \tau} \frac{\ln t_{0}}{\ln \ln t_{0}}\right), \\
& j_{1}=\int_{-H \tau / 2}^{H \tau / 2}\left(\frac{\sin u}{u}\right)^{2} S\left(t_{1}+\frac{2 u}{\tau}\right) d u \leqslant-\frac{1}{170} \sqrt{\frac{\ln H}{\ln \ln H}}+O\left(\ln \tau+\frac{1}{H \tau} \frac{\ln t_{1}}{\ln \ln t_{1}}\right) .
\end{aligned}
$$

Обозначая через $M_{0}$ и $M_{1}$ верхнюю и нижнюю грани функции $S(u)$ на отрезке $T-H \leqslant t \leqslant T+2 H$, находим

$$
\begin{aligned}
& j_{0} \leqslant M_{0} \int_{-H \tau / 2}^{H \tau / 2}\left(\frac{\sin u}{u}\right)^{2} d u<M_{0} \int_{-\infty}^{+\infty}\left(\frac{\sin u}{u}\right)^{2} d u=\frac{\pi}{2} M_{0}, \\
& j_{1} \geqslant M_{1} \int_{-H \tau / 2}^{H \tau / 2}\left(\frac{\sin u}{u}\right)^{2} d u>M_{1} \int_{-\infty}^{+\infty}\left(\frac{\sin u}{u}\right)^{2} d u=\frac{\pi}{2} M_{1} .
\end{aligned}
$$

Значит, при $r=0,1$

$$
\begin{aligned}
& (-1)^{r} M_{r}>\frac{2}{\pi}\left(\frac{1}{170} \sqrt{\frac{\ln H}{\ln \ln H}}+O\left(\ln \tau+\frac{1}{H \tau} \frac{\ln T}{\ln \ln T}\right)\right) \\
& =\frac{1}{85 \pi} \sqrt{\frac{\ln H}{\ln \ln H}}\left(1+O\left(\frac{\sqrt{\ln \ln H} \ln \ln \ln H}{\sqrt{\ln H}}\right)+O\left(\frac{\ln T}{\ln \ln T} \frac{1}{H \sqrt{\ln H} \sqrt{\ln \ln H}}\right)\right)
\end{aligned}
$$




$$
=\frac{1}{85 \pi} \sqrt{\frac{\ln H}{\ln \ln H}}\left(1+O\left(\frac{\ln \ln \ln T}{\sqrt{\ln \ln T}}\right)+O\left(\frac{1}{\sqrt{\ln \ln \ln T}}\right)\right)>\frac{1}{90 \pi} \sqrt{\frac{\ln H}{\ln \ln H}} .
$$

Теорема доказана.

ДОКАЗАТЕЛЬСТВо ТЕОРемЫ 2. Положим $H=T^{\varepsilon}, k=\left[T H^{-1} / 2\right]$. Согласно теореме 1 на каждом промежутке $(T+(2 \nu-1) H, T+2 \nu H), \nu=1,2, \ldots, k$, найдутся точки $t_{\nu}$ и $\tau_{\nu}$ такие, что

$$
S\left(t_{\nu}\right)>\frac{\sqrt{\varepsilon}}{290} \sqrt{\frac{\ln T}{\ln \ln T}}, \quad S\left(\tau_{\nu}\right)<-\frac{\sqrt{\varepsilon}}{290} \sqrt{\frac{\ln T}{\ln \ln T}} .
$$

Обозначим $h=(\ln T)^{-1}$ и покажем, что все промежутки $\left(t_{\nu}, t_{\nu}+h\right)$ содержатся во множестве $E_{0}$, а все промежутки $\left(\tau_{\nu}-h, \tau_{\nu}\right)$ - во множестве $E_{1}$.

Для этого заметим, что $S(t)$ - кусочно гладкая функция, которая терпит разрывы в точках, совпадающих с ординатами нулей $\zeta(s)$. При переходе через точку разрыва $S(t)$ совершает скачок, равный сумме кратностей нулей $\zeta(s)$, для которых эта точка явилась ординатой. По формуле Римана-Мангольдта

$$
N(t)=\frac{t}{2 \pi} \ln \frac{t}{2 \pi}-\frac{t}{2 \pi}+\frac{7}{8}+S(t)+\Delta(t)
$$

где $N(t)$ - число нулей $\zeta(s)$ в прямоугольнике $0 \leqslant \operatorname{Re} s \leqslant 1,0<\operatorname{Im} s \leqslant t$, а $\Delta(t)$ - гладкая функция, $\Delta(t) \asymp t^{-1}, \Delta^{\prime}(t) \asymp t^{-2}$. Значит, на всяком промежутке вешественной прямой, не содержашем ординат нулей $\zeta(s)$, функция $N(t)$ постоянна, а $S(t)$ является монотонно убывающей функцией с производной, равной $-\frac{1}{2 \pi} \ln \frac{t}{2 \pi}+O\left(t^{-2}\right)$. Поэтому для всех $t, t_{\nu} \leqslant t \leqslant t_{\nu}+h$, имеем

$$
S(t)>\frac{\sqrt{\varepsilon}}{290} \sqrt{\frac{\ln T}{\ln \ln T}}-h\left(\frac{1}{2 \pi} \ln \frac{t_{\nu}+h}{2 \pi}+O\left(T^{-2}\right)\right)>\frac{\sqrt{\varepsilon}}{300} \sqrt{\frac{\ln T}{\ln \ln T}},
$$

а для всех $\tau, \tau_{\nu}-h \leqslant \tau \leqslant \tau_{\nu}$,

$$
S(\tau)<-\frac{\sqrt{\varepsilon}}{290} \sqrt{\frac{\ln T}{\ln \ln T}}+h\left(\frac{1}{2 \pi} \ln \frac{\tau_{\nu}}{2 \pi}+O\left(T^{-2}\right)\right)<-\frac{\sqrt{\varepsilon}}{300} \sqrt{\frac{\ln T}{\ln \ln T}} .
$$

Эти неравенства означают, что все промежутки $\left(t_{\nu}, t_{\nu}+h\right)$ содержатся в $E_{0}$, а все промежутки $\left(\tau_{\nu}-h, \tau_{\nu}\right)-$ в $E_{1}$. Для завершения доказательства осталось заметить, что построенные промежутки попарно не пересекаются, и поэтому

$$
\operatorname{mes}\left(E_{j}\right) \geqslant k h \geqslant \frac{1}{3} T H^{-1} h=\frac{1}{3} T^{1-\varepsilon}(\ln T)^{-1}, \quad j=0,1 .
$$

Теорема доказана. 


\section{Список литературы}

1. Карацуба A. A. О функции $S(t)$ // Изв. РАН. Сер. матем. 1996. Т. 60. № 5. С. 27-56.

2. Карацуба A.A. Плотностная теорема и поведение аргумента дзета-функции Римана // Матем. заметки. 1996. Т. 60. № 3. С. 448-449.

3. Королёв M.A. О числе перемен знака функции $S(t)$ на коротком промежутке // Докл. PAH. 2002. T. 382. № 4. C. 446-447.

4. Королёв М.А. Об аргументе дзета-функции Римана на критической прямой // Тр. МИАН. 2002. Т. 239. С. 215-238.

5. Королёв М. А. Об аргументе дзета-функции Римана на критической прямой // Изв. РАН. Сер. матем. 2003. Т. 67. № 2. С. 21-60.

6. Королёв M. A. О поведении функции $S(t)$ на коротких промежутках // Докл. РАН. 2003. Т. 390. № 5. C. 588-589.

7. Королёв М. А. Поведение аргумента дзета-функции Римана на критической прямой: Автореф. дис.... канд. физ.-мат. наук. М.: МГУ, 2003.

8. Титчмари Е. К. Теория дзета-функции Римана. 1-е изд. М.: ИЛ, 1953.

9. Selberg A. Contributions to the theory of the Riemann zeta-function // Arch. Math. Naturvid. 1946. V. 48. № 5. P. 89-155.

10. Montgomery H. L. Extreme values of the Riemann zeta-function // Comment. Math. Helv. 1977. V. 52. № 4. P. 511-518.

11. Tsang K.-M. Some $\Omega$-theorems for the Riemann zeta-function // Acta Arith. 1986. V. 46. № 4. P. 369-395.

12. Ramachandra K., Sankaranarayanan A. On some theorems of Littlewood and Selberg. I // J. Number Theory. 1991. V. 44. № 3. P. 281-291.

13. Odlyzko A. M. The $10^{20}$-th zero of the Riemann zeta-function and 70 million of its neigbors. Murray Hill: AT\&T Bell Laboratories, 1987.

14. Мозер Я. О функции $S(t)$ в теории дзета-функции Римана // Acta Arith. 1976. Т. 30. № 2. C. $145-158$.

15. Ghosh A. On the Riemann zeta-function - mean value theorems and the disribution of $|S(T)|$ // J. Number Theory. 1983. V. 17. P. 93-102.

E-mail: hardy_ramanujan@mail.ru

Поступило в редакцию 10.08 .2004 